\title{
A comparison of the use of coercive measures between patients referred by the police and patients referred by other sources to a psychiatric hospital in Australia Reshin Maharaj
}

\author{
Address: Sydney West Area Health Service, 4 Norwood Place, Sydney 2153, Australia \\ from WPA Thematic Conference. Coercive Treatment in Psychiatry: A Comprehensive Review \\ Dresden, Germany. 6-8 June 2007 \\ Published: 19 December 2007 \\ BMC Psychiatry 2007, 7(SuppI I):SI27 doi:I0.II86/I47I-244X-7-SI-SI 27
}

This abstract is available from: http://www.biomedcentral.com/I47I-244X/7/SI/SI 27

(C) 2007 Maharaj; licensee BioMed Central Ltd.

\section{Background}

Police are a major source of referral to psychiatric services but the appropriateness of these referrals has been questioned. Few studies have systematically addressed police referrals to psychiatric services. This study investigated the epidemiology of coercive treatment in psychiatry by comparing the characteristics of police referrals to patients who were referred from other sources.

\section{Methods}

Data were collected from the records of 101 police referred patients and 99 patients referred from other sources. Multivariate analyses were used to test for differences between referral groups on a range of demographic, diagnostic and admission outcomes. Logistic regression was used to investigate the relative importance of predictor variables.

\section{Results}

Of the 200 patients admitted $85 \%$ were referred by involuntary legal commitment (100 police referrals and 69 from other sources). Referral groups were significantly different on a number of diagnostic and admission outcome variables. Police referrals were 3 times more likely to be diagnosed with a mental disorder due to substance misuse. Police referrals were significantly more likely to have higher total HoNOS scores (indicating higher levels of difficulties in overall functioning), experience problems with behavior and social functioning, exhibit violent behavior, be admitted to the PICU, be administered sedatives/hypnotic medications, require seclusion and spend fewer days in hospital. The most important predictor for a police referral was drug or alcohol problems.

\section{Conclusion}

The findings from this study suggest that persons referred by police to psychiatric services in Australia represent a distinct group of patients compared to patients referred by other sources. Coercive treatment measures are applied more significantly to patients referred by police but the medium-term outcome for the application of these measures is yet to be identified. 